Bull. Korean Math. Soc. 48 (2011), No. 5, pp. 923-938

http://dx.doi.org/10.4134/BKMS.2011.48.5.923

\title{
STRONG LIMIT THEOREMS FOR WEIGHTED SUMS OF NOD SEQUENCE AND EXPONENTIAL INEQUALITIES
}

\author{
Xuejun Wang, Shuhe Hu, And Andrei I. Volodin
}

\begin{abstract}
Some properties for negatively orthant dependent sequence are discussed. Some strong limit results for the weighted sums are obtained, which generalize the corresponding results for independent sequence and negatively associated sequence. At last, exponential inequalities for negatively orthant dependent sequence are presented.
\end{abstract}

\section{Introduction}

Recently, $\mathrm{Wu}[11]$ proved the equivalence of the a.s. and complete convergence for weighted sum $\sum_{i=1}^{n} X_{i} /((n+2-i) \log (n+2-i) \log \log n)$ of independent and identically distributed random variables. Antonini et al. [2] gave some conditions on weights so that the weighted sum converges completely to zero, which improved the theorem of Chow and Lai [4] and extended the theorem of $\mathrm{Wu}[11]$ to the more general weighted sums.

The main purpose of the paper is to extend the results for weighted sums of independent and identically distributed random variables to the case of negatively orthant dependent random variables. The techniques involved with the main results are inspired by Adler [1] and Antonini et al. [2].

Some definitions and lemmas are needed.

Definition 1.1. A finite collection of random variables $X_{1}, X_{2}, \ldots, X_{n}$ is said to be negatively associated (NA) if for every pair of disjoint subsets $A_{1}, A_{2}$ of $\{1,2, \ldots, n\}$,

$$
\operatorname{Cov}\left\{f\left(X_{i}: i \in A_{1}\right), g\left(X_{j}: j \in A_{2}\right)\right\} \leq 0,
$$

Received November 20, 2009; Revised July 1, 2010.

2010 Mathematics Subject Classification. 60F15, 60E15.

Key words and phrases. strong convergence, negatively associated random variables, negatively orthant dependent random variables, exponential inequality.

Supported by the NNSF of China (10871001), Provincial Natural Science Research Project of Anhui Colleges (KJ2010A005), Talents Youth Fund of Anhui Province Universities (2010SQRL016ZD), Youth Science Research Fund of Anhui University (2009QN011A) and the Academic innovation team of Anhui University (KJTD001B). 
whenever $f$ and $g$ are coordinatewise nondecreasing such that this covariance exists. An infinite sequence $\left\{X_{n}, n \geq 1\right\}$ is NA if every finite subcollection is NA.

Definition 1.2. A finite collection of random variables $X_{1}, X_{2}, \ldots, X_{n}$ is said to be negatively upper orthant dependent (NUOD) if for all real numbers $x_{1}, x_{2}, \ldots, x_{n}$,

$$
P\left(X_{i}>x_{i}, i=1,2, \ldots, n\right) \leq \prod_{i=1}^{n} P\left(X_{i}>x_{i}\right),
$$

and negatively lower orthant dependent (NLOD) if for all real numbers $x_{1}, x_{2}$, $\ldots, x_{n}$,

$$
P\left(X_{i} \leq x_{i}, i=1,2, \ldots, n\right) \leq \prod_{i=1}^{n} P\left(X_{i} \leq x_{i}\right) .
$$

A finite collection of random variables $X_{1}, X_{2}, \ldots, X_{n}$ is said to be negatively orthant dependent (NOD) if they are both NUOD and NLOD. An infinite sequence $\left\{X_{n}, n \geq 1\right\}$ is said to be NOD if every finite subcollection is NOD.

The concepts of NA and NOD sequences were introduced by Joag-Dev and Proschan [6]. Obviously, independent random variables are NOD. Joag-Dev and Proschan [6] pointed out that NA random variables are NOD, but neither NUOD nor NLOD implies NA. They also presented an example in which $X=$ $\left(X_{1}, X_{2}, X_{3}, X_{4}\right)$ possesses NOD, but does not possess NA. So we can see that NOD is weaker than NA. For more details about NOD random variables, one can refer to Ko and Kim [9], Fakoor and Azarnoosh [5], Ko et al. [8], Kim [7], $\mathrm{Wu}[10]$, and so forth.

Throughout the paper, let $\left\{X_{n}, n \geq 1\right\}$ be a sequence of NOD random variables defined on a fixed probability space $(\Omega, \mathcal{F}, P)$. Let $a_{n} \ll b_{n}$ denote that there exists a constant $C>0$ such that $\left|a_{n} / b_{n}\right| \leq C$ for sufficiently large $n$. Denote $X^{+} \doteq \max (0, X), X^{-} \doteq \max (0,-X)$ and $\log n=\ln n$. $C$ denotes a positive constant which may be different in various places. The main results of this paper are depending on the following lemmas:

Lemma 1.1 (cf. Bozorgnia, et al., [3]). Let random variables $X_{1}, X_{2}, \ldots, X_{n}$ be NOD, $f_{1}, f_{2}, \ldots, f_{n}$ be all nondecreasing (or nonincreasing) functions. Then random variables $f_{1}\left(X_{1}\right), f_{2}\left(X_{2}\right), \ldots, f_{n}\left(X_{n}\right)$ are NOD.

Lemma 1.2 (cf. Bozorgnia, et al., [3]). Let random variables $X_{1}, X_{2}, \ldots, X_{n}$ be nonnegatively NOD. Then

$$
E\left(\prod_{j=1}^{n} X_{j}\right) \leq \prod_{j=1}^{n} E\left(X_{j}\right)
$$


Lemma 1.3 (cf. Kim, [7]). Let $X_{1}, X_{2}, \ldots, X_{n}$ be NOD random variables with $E X_{n}=0$ and $E X_{n}^{2}<\infty$ for all $n \geq 1$. Then we have

(1.5) $E\left(X_{m+1}+X_{m+2}+\cdots+X_{m+p}\right)^{2} \leq E X_{m+1}^{2}+E X_{m+2}^{2}+\cdots+E X_{m+p}^{2}$

for all integers $m \geq 0, p \geq 1$ and $m+p \leq n$. Moreover, we have

$$
E\left(\max _{1 \leq k \leq n}\left|\sum_{i=1}^{k} X_{i}\right|^{2}\right) \leq\left(\log _{3} n+2\right)^{2} \sum_{i=1}^{n} E X_{i}^{2} .
$$

By Lemma 1.1 and Lemma 1.3, we can get the following Khintchine-Kolmogorov type convergence theorem and three series theorem for NOD sequences, which can be applied to obtain the main results of the paper. The proofs are standard, so we omit them.

Corollary 1.1 (Khintchine-Kolmogorov-type convergence theorem). Let $\left\{X_{n}\right.$, $n \geq 1\}$ be a sequence of NOD random variables. If

$$
\sum_{n=1}^{\infty} \operatorname{Var}\left(X_{n}\right) \log ^{2} n<\infty
$$

then $\sum_{n=1}^{\infty}\left(X_{n}-E X_{n}\right)$ converges a.s..

Corollary 1.2 (Three series theorem for NOD sequence). Let $\left\{X_{n}, n \geq 1\right\}$ be a sequence of NOD random variables. Assume that for some $a>0$

$$
\begin{aligned}
& \sum_{n=1}^{\infty} P\left(\left|X_{n}\right|>a\right)<\infty, \\
& \sum_{n=1}^{\infty} E X_{n}^{(a)} \text { converges, } \\
& \sum_{n=1}^{\infty} \operatorname{Var}\left(X_{n}^{(a)}\right) \log ^{2} n<\infty .
\end{aligned}
$$

Then $\sum_{n=1}^{\infty} X_{n}$ converges a.s., where $X_{n}^{(a)}=-a I\left(X_{n}<-a\right)+X_{n} I\left(\left|X_{n}\right| \leq\right.$ a) $+a I\left(X_{n}>a\right)$.

The organization of this paper is as follows. Some properties for NOD sequence are provided in Section 2 and strong limit results for weighted sums of NOD sequence are given in Section 3. An exponential inequality for NOD sequence is proved in Section 4. 


\section{Properties for NOD sequence}

In this section, we will present some propositions for NOD sequence, which can be applied to prove some of the main results of the paper.

Proposition 2.1. Let $\left\{X_{n}, n \geq 1\right\}$ be a sequence of NOD random variables and $\left\{x_{n}, n \geq 1\right\}$ be a sequence of real numbers. Denote $E_{i}=\left(X_{i} \leq x_{i}\right)$, $i=1,2, \ldots$ or $E_{i}=\left(X_{i}>x_{i}\right), i=1,2, \ldots$ Then

$$
P\left(\bigcap_{k=1}^{\infty} E_{k}\right) \leq \prod_{k=1}^{\infty} P\left(E_{k}\right) .
$$

Proof. By the continuity of probability and the definition of NOD, we can get

$$
P\left(\bigcap_{k=1}^{\infty} E_{k}\right)=\lim _{n \rightarrow \infty} P\left(\bigcap_{k=1}^{n} E_{k}\right) \leq \lim _{n \rightarrow \infty} \prod_{k=1}^{n} P\left(E_{k}\right)=\prod_{k=1}^{\infty} P\left(E_{k}\right) .
$$

The proof is completed.

Proposition 2.2. Let $\left\{X_{n}, n \geq 1\right\}$ be a sequence of NOD random variables and $\left\{x_{n}, n \geq 1\right\}$ be a sequence of real numbers. Denote $E_{i}=\left(X_{i} \leq x_{i}\right)$, $i=1,2, \ldots$, or $E_{i}=\left(X_{i}>x_{i}\right), i=1,2, \ldots$ Then $\sum_{n=1}^{\infty} P\left(E_{n}\right)=\infty$ implies $P\left(E_{n}\right.$, i.o. $)=1$.

Proof. By the continuity of probability, Proposition 2.1 and $\sum_{n=1}^{\infty} P\left(E_{n}\right)=\infty$, we have

$$
\begin{aligned}
0 \leq 1-P\left(E_{n}, \text { i.o. }\right) & =\lim _{n \rightarrow \infty} P\left(\bigcap_{k=n}^{\infty} \bar{E}_{k}\right) \\
& \leq \lim _{n \rightarrow \infty} \prod_{k=n}^{\infty} P\left(\bar{E}_{k}\right) \\
& \leq \lim _{n \rightarrow \infty} \prod_{k=n}^{\infty} e^{-P\left(E_{k}\right)}=0 .
\end{aligned}
$$

Therefore, $P\left(E_{n}\right.$, i.o. $)=1$.

Proposition 2.3. Under the conditions of Proposition 2.2,

$$
\sum_{n=1}^{\infty} P\left(E_{n}\right)=\infty \Leftrightarrow P\left(E_{n}, i . o .\right)=1 .
$$

Proposition 2.4. Let $\left\{X_{n}, n \geq 1\right\}$ be a sequence of NOD random variables. Then

$$
X_{n} \rightarrow 0 \text { a.s. } \Leftrightarrow \sum_{n=1}^{\infty} P\left(\left|X_{n}\right|>\varepsilon\right)<\infty \text { for any } \varepsilon>0
$$


Proof. " $\Leftarrow "$ If $\sum_{n=1}^{\infty} P\left(\left|X_{n}\right|>\varepsilon\right)<\infty$ for any $\varepsilon>0$, then $X_{n} \rightarrow 0$ a.s. follows from Borel-Cantelli Lemma immediately.

" $\Rightarrow$ " Let $X_{n} \rightarrow 0$ a.s., we can see that $X_{n}^{+} \rightarrow 0$ a.s., $X_{n}^{-} \rightarrow 0$ a.s. Denote

$$
E_{n}(1)=\left(X_{n}^{+}>\varepsilon / 2\right), E_{n}(2)=\left(X_{n}^{-}>\varepsilon / 2\right) \text { for any } \varepsilon>0 \text {, }
$$

thus

$$
P\left(E_{n}(j), \text { i.o. }\right)=0, j=1,2 .
$$

By Lemma 1.1, we can see that $\left\{X_{n}^{+}, n \geq 1\right\}$ and $\left\{X_{n}^{-}, n \geq 1\right\}$ are both NOD. By Proposition 2.2 and (2.2),

$$
\sum_{n=1}^{\infty} P\left(E_{n}(j)\right)<\infty, j=1,2 .
$$

Therefore,

$$
\sum_{n=1}^{\infty} P\left(\left|X_{n}\right|>\varepsilon\right) \leq \sum_{n=1}^{\infty} P\left(X_{n}^{+}>\varepsilon / 2\right)+\sum_{n=1}^{\infty} P\left(X_{n}^{-}>\varepsilon / 2\right)<\infty .
$$

The proof is completed.

Proposition 2.5. Let $\left\{X_{n}, n \geq 1\right\}$ be a sequence of NOD random variables and $\left\{x_{n}, n \geq 1\right\}$ be a sequence of positive numbers. Denote $G_{i}=\left(\left|X_{i}\right|>x_{i}\right)$ for $i=1,2, \ldots$ Then $\sum_{n=1}^{\infty} P\left(G_{n}\right)=\infty$ implies $P\left(G_{n}\right.$, i.o. $)=1$.

Proof. Denote $E_{i}=\left(X_{i}>x_{i}\right)$ and $F_{i}=\left(X_{i}<-x_{i}\right)$ for $i=1,2, \ldots$ It is easily seen that $G_{i}=E_{i}+F_{i}$. Thus, $\sum_{n=1}^{\infty} P\left(G_{n}\right)=\infty$ implies that

$$
\sum_{n=1}^{\infty} P\left(E_{n}\right)=\infty, \text { or } \sum_{n=1}^{\infty} P\left(F_{n}\right)=\infty \text {. }
$$

By (2.5) and Proposition 2.2, we have $P\left(E_{n}\right.$, i.o. $)=1$ or $P\left(F_{n}\right.$, i.o. $)=1$, which implies that $P\left(G_{n}\right.$, i.o. $)=1$.

Proposition 2.6. Under the conditions of Proposition 2.5,

$$
\sum_{n=1}^{\infty} P\left(G_{n}\right)=\infty \Leftrightarrow P\left(G_{n}, \text { i.o. }\right)=1 \text {. }
$$

\section{Strong limit results for the weighted sums of NOD sequence}

In this section, we will provide some strong limit results for the weighted sums of NOD sequence and their proofs, which extend the results of Antonini et al. [2].

Theorem 3.1. Let $\left\{X_{n}, n \geq 1\right\}$ be a sequence of NOD random variables with identical distribution, $E X_{1}=0$ and $E\left[e^{t\left|X_{1}\right|}\right]<\infty$ for all $t>0$. Let $\left\{a_{n i}, n \geq\right.$ $\left.1,1 \leq i \leq m_{n}\right\}$ be an array of positive constants satisfying the following two conditions, where $\left\{m_{n}, n \geq 1\right\}$ is a sequence of positive integers:

(i) $\max _{1 \leq i \leq m_{n}} a_{n i} \log n=\bar{O}(1)$, 
(ii) $\sum_{i=1}^{m_{n}} a_{n i}^{2} \log n=o(1)$.

Then $\sum_{i=1}^{i=1} a_{n} a_{n i} X_{i}$ converges completely to zero, which implies that $\sum_{i=1}^{m_{n}} a_{n i} X_{i}$ converges almost surely to zero.

Proof. The proof is inspired by Theorem 1 of Antonini et al. [2]. It can be checked that for all $x \in \mathbf{R}$, the following inequality holds

$$
e^{x} \leq 1+x+\frac{1}{2} x^{2} e^{|x|}
$$

thus, by $E X_{n}=0$, we have

$$
E\left[e^{t a_{n i} X_{i}}\right] \leq 1+E\left[\frac{1}{2} t^{2} a_{n i}^{2} X_{1}^{2} e^{t a_{n i}\left|X_{1}\right|}\right]
$$

for any $t>0$. Let $\varepsilon>0$ be given. If we take $t=2 \log n / \varepsilon$, then we can obtain

$$
\begin{aligned}
E\left[e^{t a_{n i} X_{i}}\right] & \leq 1+\frac{1}{2}\left(\frac{2}{\varepsilon}\right)^{2} \log ^{2} n a_{n i}^{2} E\left[X_{1}^{2} e^{\frac{2}{\varepsilon} \log n a_{n i}\left|X_{1}\right|}\right] \\
& \leq 1+\frac{1}{2}\left(\frac{2}{\varepsilon}\right)^{2} \log ^{2} n a_{n i}^{2} E\left[X_{1}^{2} e^{C\left|X_{1}\right|}\right] \\
& \leq 1+\frac{1}{2}\left(\frac{2}{\varepsilon}\right)^{2} \log ^{2} n a_{n i}^{2} E\left[e^{(1+C)\left|X_{1}\right|}\right]
\end{aligned}
$$

following from $x^{2} \leq e^{|x|}$ for all $x \in \mathbf{R}$. Since $E\left[e^{t\left|X_{1}\right|}\right]<\infty$ for all $t>0$, we have

$$
E\left[e^{t a_{n i} X_{i}}\right] \leq 1+C a_{n i}^{2} \log ^{2} n
$$

By Lemma 1.1, Lemma 1.2 and (3.2),

$$
\begin{aligned}
P\left(\sum_{i=1}^{m_{n}} a_{n i} X_{i}>\varepsilon\right) & \leq e^{-t \varepsilon} E\left(e^{t \sum_{i=1}^{m_{n}} a_{n i} X_{i}}\right)=e^{-t \varepsilon} E\left(\prod_{i=1}^{m_{n}} e^{t a_{n i} X_{i}}\right) \\
& \leq e^{-2 \log n} \prod_{i=1}^{m_{n}}\left(1+C a_{n i}^{2} \log ^{2} n\right) \\
& \leq e^{-2 \log n} \prod_{i=1}^{m_{n}} e^{C a_{n i}^{2} \log ^{2} n} \leq e^{-\frac{3}{2} \log n}=n^{-\frac{3}{2}}
\end{aligned}
$$

for all sufficiently large $n$. The last inequality follows from the condition (ii). According to Lemma 1.1, we can see that $\left\{-X_{n}, n \geq 1\right\}$ is also negatively orthant dependent with identical distribution, $E\left(-X_{n}\right)=0$ and $E\left[e^{t\left|-X_{1}\right|}\right]<$ $\infty$ for all $t>0$. Replace $X_{i}$ by $-X_{i}$ from the above statement, we obtain

$$
P\left(\sum_{i=1}^{m_{n}} a_{n i} X_{i}<-\varepsilon\right)=P\left(\sum_{i=1}^{m_{n}} a_{n i}\left(-X_{i}\right)>\varepsilon\right) \leq n^{-\frac{3}{2}}
$$


for all sufficiently large $n$. By (3.3) and (3.4), it follows that

$$
\begin{aligned}
& \sum_{n=1}^{\infty} P\left(\left|\sum_{i=1}^{m_{n}} a_{n i} X_{i}\right|>\varepsilon\right) \\
\leq & \sum_{n=1}^{\infty} P\left(\sum_{i=1}^{m_{n}} a_{n i} X_{i}>\varepsilon\right)+\sum_{n=1}^{\infty} P\left(\sum_{i=1}^{m_{n}} a_{n i} X_{i}<-\varepsilon\right) \\
\leq & C \sum_{n=1}^{\infty} n^{-\frac{3}{2}}<\infty .
\end{aligned}
$$

Thus, $\sum_{i=1}^{m_{n}} a_{n i} X_{i}$ converges completely to zero. We get the desired result.

Corollary 3.1. Let $\left\{X_{n}, n \geq 1\right\}$ be a sequence of NOD random variables with identical distribution, $E X_{1}=0$ and $E\left[e^{t\left|X_{1}\right|}\right]<\infty$ for all $t>0$. Let $\left\{c_{n i}, n \geq\right.$ $1,1 \leq i \leq n\}$ be an array of positive constants such that $\lim _{\sup _{n \rightarrow \infty}} \sum_{i=1}^{n} c_{n i}^{2}<$ $\infty$. Then $\sum_{i=1}^{n} c_{n i} X_{i} / \log n$ converges completely to zero.

Proof. Let $a_{n i}=c_{n i} / \log n$, we can easily get the desired result by Theorem 3.1.

The next theorem examines what happens when $P\left(\left|X_{n}\right|>c_{n}\right.$, i.o. $)=1$.

Theorem 3.2. Let $\left\{X_{n}, n \geq 1\right\}$ be a sequence of NOD random variables with identical distribution

$$
P\left(\left|X_{1}\right|>x\right)=\left\{\begin{array}{cc}
L(x) x^{-\alpha}, & x \geq 1 \\
1, & x<1
\end{array}\right.
$$

where $L(x)$ is a slowly varying function (i.e., $L(x)$ is a positive function defined on $[0,+\infty)$ and $L(c x) / L(x) \rightarrow 1$ as $x \rightarrow \infty$ for all $c>0), \alpha \geq 0$. Let $\left\{a_{n}, n \geq\right.$ $1\}$ and $\left\{b_{n}, n \geq 1\right\}$ be sequences of positive constants satisfying $b_{n}=O\left(b_{n+1}\right)$ and $b_{n} \rightarrow \infty$. Denote $c_{n}=b_{n} / a_{n}$ and $S_{n}=\sum_{i=1}^{n} a_{i} X_{i}$ for each $n \geq 1$. Assume that

$$
\sum_{n=1}^{\infty} P\left(\left|X_{n}\right|>c_{n}\right)=\infty
$$

then

$$
\limsup _{n \rightarrow \infty} \frac{\left|S_{n}\right|}{b_{n}}=\infty \text { a.s.. }
$$

Proof. If $c_{n} \rightarrow \infty$, then for all sufficiently large $M$,

$$
\begin{aligned}
\sum_{n=1}^{\infty} P\left(\left|a_{n} X_{n}\right|>M b_{n}\right) & =\sum_{n=1}^{\infty} L\left(M c_{n}\right)\left(M c_{n}\right)^{-\alpha} \geq C \sum_{n=n_{0}}^{\infty} L\left(c_{n}\right) c_{n}^{-\alpha} \\
& =C \sum_{n=n_{0}}^{\infty} P\left(\left|X_{n}\right|>c_{n}\right)=\infty
\end{aligned}
$$

for a suitable integer $n_{0}$ such that $c_{n} \geq 1$ for all $n \geq n_{0}$. 
On the other hand, if $\liminf _{n \rightarrow \infty} c_{n}<\infty$, then there exist a subsequence $\left\{n_{k}, k \geq 1\right\}$ and a finite positive constant $B$ such that $c_{n_{k}} \leq B$. Hence for all $0<M<\infty$

$$
\begin{aligned}
\sum_{n=1}^{\infty} P\left(\left|a_{n} X_{n}\right|>M b_{n}\right) & =\sum_{n=1}^{\infty} P\left(\left|X_{n}\right|>M c_{n}\right) \geq \sum_{k=1}^{\infty} P\left(\left|X_{1}\right|>M c_{n_{k}}\right) \\
& \geq \sum_{k=1}^{\infty} P\left(\left|X_{1}\right|>M B\right)=\infty
\end{aligned}
$$

In both cases, by Proposition 2.5 or Proposition 2.6, we can get

$$
\limsup _{n \rightarrow \infty}\left|\frac{a_{n} X_{n}}{b_{n}}\right|=\infty \text { a.s.. }
$$

Since

$$
\left|\frac{a_{n} X_{n}}{b_{n}}\right| \leq\left|\frac{S_{n}}{b_{n}}\right|+\left|\frac{b_{n-1}}{b_{n}}\right| \cdot\left|\frac{S_{n-1}}{b_{n-1}}\right|,
$$

the desired result (3.6) follows from (3.7) and (3.8) immediately.

Theorem 3.3. Let $\left\{X_{n}, n \geq 1\right\}$ be a sequence of mean zero NOD random variables with identical distribution (3.5) and $1<\alpha<2$. Let $\left\{a_{n}, n \geq 1\right\}$ and $\left\{b_{n}, n \geq 1\right\}$ be sequences of positive constants satisfying $0<b_{n} \uparrow \infty$. Denote $c_{1}=b_{1} / a_{1}$ and $c_{n}=b_{n} /\left(a_{n} \log n\right)$ for $n \geq 2$. Assume that

$$
\sum_{n=1}^{\infty} P\left(\left|X_{n}\right|>c_{n}\right)<\infty
$$

then

$$
\frac{1}{b_{n}} \sum_{k=1}^{n} a_{k} X_{k} \rightarrow 0 \text { a.s. }
$$

Proof. (3.5) and (3.9) imply that $c_{k} \geq 1$ for all sufficiently large $k$. Without loss of generality, we assume that $c_{k} \geq 1$ for all $k \geq 1$.

By Borel-Cantelli Lemma, it is easily seen that (3.9) implies that

$$
\sum_{k=1}^{n} a_{k} X_{k} I\left(\left|X_{k}\right|>c_{k}\right)=o\left(b_{n}\right) \text { a.s. }
$$

Denote

$$
Y_{k}=-c_{k} I\left(X_{k}<-c_{k}\right)+X_{k} I\left(\left|X_{k}\right| \leq c_{k}\right)+c_{k} I\left(X_{k}>c_{k}\right), k \geq 1,
$$

then $\left\{Y_{k}, k \geq 1\right\}$ are still NOD from Lemma 1.1. It is easy to check that $\sum_{k=1}^{n} a_{k} X_{k}=\sum_{k=1}^{n} a_{k}\left(Y_{k}-E Y_{k}\right)+\sum_{k=1}^{n} a_{k} E Y_{k}+\sum_{k=1}^{n} a_{k} c_{k}\left(I\left(X_{k}<-c_{k}\right)-I\left(X_{k}>c_{k}\right)\right)$ 


$$
+\sum_{k=1}^{n} a_{k} X_{k} I\left(\left|X_{k}\right|>c_{k}\right)
$$

In order to show $\frac{1}{b_{n}} \sum_{k=1}^{n} a_{k} X_{k} \rightarrow 0$ a.s., we only need to show that the first three terms above are $o\left(b_{n}\right)$ or $o\left(b_{n}\right)$ a.s..

By $C_{r}$ inequality, Theorem $1 \mathrm{~b}$ in Feller $(1971, \mathrm{p} .281)$ and $(3.9)$, we can get

$$
\begin{aligned}
\sum_{k=1}^{\infty} \log ^{2} k \operatorname{Var}\left(\frac{a_{k} Y_{k}}{b_{k}}\right) & \leq C \sum_{k=1}^{\infty} c_{k}^{-2} E Y_{k}^{2} \\
& \leq C \sum_{k=1}^{\infty} c_{k}^{-2} E\left[c_{k}^{2} I\left(\left|X_{k}\right|>c_{k}\right)+X_{k}^{2} I\left(\left|X_{k}\right| \leq c_{k}\right)\right] \\
& =C \sum_{k=1}^{\infty} P\left(\left|X_{k}\right|>c_{k}\right)+C \sum_{k=1}^{\infty} c_{k}^{-2} E X_{k}^{2} I\left(\left|X_{k}\right| \leq c_{k}\right) \\
& \leq C+C \sum_{k=1}^{\infty} c_{k}^{-2} \int_{0}^{c_{k}} t P\left(\left|X_{k}\right|>t\right) d t \\
& \leq C+C \sum_{k=1}^{\infty} L\left(c_{k}\right) c_{k}^{-\alpha} \\
& \leq C+C \sum_{k=1}^{\infty} P\left(\left|X_{k}\right|>c_{k}\right)<\infty
\end{aligned}
$$

By Corollary 1.1 and Kronecker's Lemma, we have

$$
\sum_{k=1}^{n} a_{k}\left(Y_{k}-E Y_{k}\right)=o\left(b_{n}\right) \text { a.s.. }
$$

By (3.9) again,

$$
\begin{aligned}
& \sum_{k=1}^{\infty} E\left|\frac{a_{k}(\log k) c_{k}\left(I\left(X_{k}<-c_{k}\right)-I\left(X_{k}>c_{k}\right)\right)}{b_{k}}\right| \\
\leq & \sum_{k=1}^{\infty} E\left(I\left(X_{k}<-c_{k}\right)+I\left(X_{k}>c_{k}\right)\right) \\
= & \sum_{k=1}^{\infty} P\left(\left|X_{k}\right|>c_{k}\right)<\infty
\end{aligned}
$$

which implies that

$$
\sum_{k=1}^{\infty} \frac{a_{k} c_{k}\left(I\left(X_{k}<-c_{k}\right)-I\left(X_{k}>c_{k}\right)\right)}{b_{k}} \text { converges a.s.. }
$$


By Kronecker's Lemma, it follows that

$$
\sum_{k=1}^{n} a_{k} c_{k}\left(I\left(X_{k}<-c_{k}\right)-I\left(X_{k}>c_{k}\right)\right)=o\left(b_{n}\right) \text { a.s.. }
$$

By Theorem 1a in Feller (1971, p.281) and (3.9) again, we have

$$
\begin{aligned}
\sum_{k=1}^{\infty}\left|\frac{a_{k}(\log k) E Y_{k}}{b_{k}}\right| & \leq \sum_{k=1}^{\infty} c_{k}^{-1}\left[c_{k} P\left(\left|X_{k}\right|>c_{k}\right)+E\left|X_{k}\right| I\left(\left|X_{k}\right|>c_{k}\right)\right] \\
& =\sum_{k=1}^{\infty} P\left(\left|X_{k}\right|>c_{k}\right)+\sum_{k=1}^{\infty} c_{k}^{-1} E\left|X_{k}\right| I\left(\left|X_{k}\right|>c_{k}\right) \\
& =2 \sum_{k=1}^{\infty} P\left(\left|X_{k}\right|>c_{k}\right)+\sum_{k=1}^{\infty} c_{k}^{-1} \int_{c_{k}}^{\infty} P\left(\left|X_{k}\right|>t\right) d t \\
& \leq C+C \sum_{k=1}^{\infty} c_{k}^{-1} \int_{c_{k}}^{\infty} L(t) t^{-\alpha} d t \leq C+C \sum_{k=1}^{\infty} L\left(c_{k}\right) c_{k}^{-\alpha} \\
& \leq C+C \sum_{k=1}^{\infty} P\left(\left|X_{k}\right|>c_{k}\right)<\infty,
\end{aligned}
$$

which implies that

$$
\sum_{k=1}^{\infty} \frac{a_{k} E Y_{k}}{b_{k}} \text { converges. }
$$

By Kronecker's Lemma, it follows that

$$
\sum_{k=1}^{n} a_{k} E Y_{k}=o\left(b_{n}\right)
$$

Hence, the desired result (3.10) follows from (3.11)-(3.15) immediately. The proof is completed.

By Theorem 3.2 and Theorem 3.3, we can get the following result.

Theorem 3.4. Let $\left\{X_{n}, n \geq 1\right\}$ be a sequence of NOD random variables with identical distribution (3.5) and $E X_{1}=0$. Let $\left\{a_{n}, n \geq 1\right\}$ and $\left\{b_{n}, n \geq 1\right\}$ be sequences of positive constants satisfying $0<b_{n} \uparrow \infty$. Denote $c_{1}=b_{1} / a_{1}$ and $c_{n}=b_{n} /\left(a_{n} \log n\right)$ for $n \geq 2$. Then for all $\alpha \in(1,2)$,

$$
\limsup _{n \rightarrow \infty}\left|\frac{1}{b_{n}} \sum_{k=1}^{n} a_{k} X_{k}\right|=0 \text { or } \infty \text { a.s. }
$$

depending on whether $\sum_{k=1}^{\infty} P\left(\left|X_{k}\right|>c_{k}\right)$ converges or $\sum_{k=1}^{\infty} P\left(\left|X_{k}\right|>b_{k} / a_{k}\right)$ diverges.

Theorem 3.5. Let $\left\{X_{n}, n \geq 1\right\}$ be a sequence of NOD random variables with identical distribution and $\left\{a_{n}, n \geq 1\right\}$ be a sequence of positive numbers with 
$A_{n} \doteq \sum_{j=1}^{n} a_{j} \uparrow \infty .1 \leq r<2$. Denote $c_{1}=1$ and $c_{n}=A_{n} /\left(a_{n} \log n\right)$ for $n \geq 2$. Assume that

$$
\begin{gathered}
E X_{1}=0, E\left|X_{1}\right|^{r}<\infty, \\
N(n) \doteq \operatorname{Card}\left\{i: c_{i} \leq n\right\} \ll n^{r}, n \geq 1,
\end{gathered}
$$

then

$$
A_{n}^{-1} \sum_{i=1}^{n} a_{i} X_{i} \rightarrow 0 \text { a.s.. }
$$

Proof. Let $N(0)=0$ and

$$
Y_{n}=-c_{n} I\left(X_{n}<-c_{n}\right)+X_{n} I\left(\left|X_{n}\right| \leq c_{n}\right)+c_{n} I\left(X_{n}>c_{n}\right), n \geq 1 .
$$

By (3.18), it is easily seen that $c_{n} \rightarrow \infty$ as $n \rightarrow \infty$ (otherwise, there exist infinite subscripts $i$ and some $n_{0}$ such that $c_{i} \leq n_{0}^{r}$, hence, $N\left(n_{0}\right)=\infty$, which is contrary to $N\left(n_{0}\right) \ll n_{0}^{r}$ from (3.18)). By (3.17) and (3.18), it follows that

$$
\begin{aligned}
\sum_{i=1}^{\infty} P\left(X_{i} \neq Y_{i}\right) & =\sum_{i=1}^{\infty} P\left(\left|X_{i}\right|>c_{i}\right)=\sum_{i=1}^{\infty} \sum_{c_{i} \leq j<c_{i}+1} P\left(\left|X_{i}\right|>c_{i}\right) \\
& \leq \sum_{j=1}^{\infty} \sum_{j-1<c_{i} \leq j} P\left(\left|X_{1}\right|>j-1\right) \\
& =\sum_{j=1}^{\infty}(N(j)-N(j-1)) P\left(\left|X_{1}\right|>j-1\right) \\
& =\sum_{j=1}^{\infty}(N(j)-N(j-1)) \sum_{l=j}^{\infty} P\left(l-1<\left|X_{1}\right| \leq l\right) \\
& =\sum_{l=1}^{\infty} \sum_{j=1}^{l}(N(j)-N(j-1)) P\left(l-1<\left|X_{1}\right| \leq l\right) \\
& =\sum_{l=1}^{\infty} N(l) P\left(l-1<\left|X_{1}\right| \leq l\right) \\
& \ll \sum_{l=1}^{\infty} l^{r} P\left(l-1<\left|X_{1}\right| \leq l\right) \ll E\left|X_{1}\right|^{r}<\infty .
\end{aligned}
$$

By the above inequality and Borel-Cantelli Lemma, we can see that $P\left(X_{i} \neq\right.$ $Y_{i}$, i.o. $)=0$. Therefore, in order to prove (3.19), we only need to prove

$$
A_{n}^{-1} \sum_{i=1}^{n} a_{i} Y_{i} \rightarrow 0 \text { a.s.. }
$$


By (3.17) and (3.18) again,

$$
\begin{aligned}
& \sum_{i=1}^{\infty} \log ^{2} i \operatorname{Var}\left(\frac{a_{i} Y_{i}}{A_{i}}\right) \\
& \leq \sum_{i=1}^{\infty} c_{i}^{-2} E Y_{i}^{2} \\
& \leq C \sum_{i=1}^{\infty} P\left(\left|X_{i}\right|>c_{i}\right)+C \sum_{i=1}^{\infty} c_{i}^{-2} E X_{1}^{2} I\left(\left|X_{1}\right| \leq c_{i}\right) \\
& \leq C+C \sum_{j=1}^{\infty} \sum_{j-1<c_{i} \leq j} c_{i}^{-2} E X_{1}^{2} I\left(\left|X_{1}\right| \leq c_{i}\right) \\
& \leq C+C \sum_{j=1}^{\infty} \sum_{j-1<c_{i} \leq j} c_{i}^{-2} E X_{1}^{2} I\left(\left|X_{1}\right| \leq j\right) \\
& \ll C+\sum_{j=2}^{\infty}(N(j)-N(j-1))(j-1)^{-2} \sum_{k=1}^{j} E X_{1}^{2} I\left(k-1<\left|X_{1}\right| \leq k\right) \\
& \ll C+\sum_{k=2}^{\infty} \sum_{j=k}^{\infty}(N(j)-N(j-1))(j-1)^{-2} E X_{1}^{2} I\left(k-1<\left|X_{1}\right| \leq k\right) \\
& \ll C+\sum_{k=2}^{\infty} \sum_{j=k}^{\infty} N(j)\left((j-1)^{-2}-j^{-2}\right) E X_{1}^{2} I\left(k-1<\left|X_{1}\right| \leq k\right) \\
& \ll C+\sum_{k=2}^{\infty} \sum_{j=k}^{\infty} j^{r-3} E X_{1}^{2} I\left(k-1<\left|X_{1}\right| \leq k\right) \\
& \ll C+\sum_{k=2}^{\infty} k^{r-2} E\left|X_{1}\right|^{r} k^{2-r} I\left(k-1<\left|X_{1}\right| \leq k\right) \\
& =C+\sum_{k=2}^{\infty} E\left|X_{1}\right|^{r} I\left(k-1<\left|X_{1}\right| \leq k\right) \\
& \ll C+E\left|X_{1}\right|^{r}<\infty \text {. }
\end{aligned}
$$

Therefore, by the above inequality, Corollary 1.1 and Kronecker's Lemma, we have

$$
A_{n}^{-1} \sum_{i=1}^{n} a_{i}\left(Y_{i}-E Y_{i}\right) \rightarrow 0 \text { a.s. }
$$

In order to prove $(3.20)$, it suffices to prove that

$$
A_{n}^{-1} \sum_{i=1}^{n} a_{i} E Y_{i} \rightarrow 0, n \rightarrow \infty .
$$


It is easily seen that $E\left|X_{1}\right|^{r}<\infty$ for $1 \leq r<2$ implies that $E\left|X_{1}\right|<\infty$, thus

$$
\lim _{i \rightarrow \infty} c_{i} P\left(\left|X_{i}\right|>c_{i}\right)=0 .
$$

By Lebesgue Dominated Convergence Theorem and $E X_{1}=0$, we have

$$
E X_{i} I\left(\left|X_{i}\right| \leq c_{i}\right)=E X_{1} I\left(\left|X_{1}\right| \leq c_{i}\right) \rightarrow E X_{1}=0, i \rightarrow \infty .
$$

Therefore,

$$
\left|E Y_{i}\right| \leq c_{i} P\left(\left|X_{i}\right|>c_{i}\right)+\left|E X_{i} I\left(\left|X_{i}\right| \leq c_{i}\right)\right| \rightarrow 0 \text { as } i \rightarrow \infty,
$$

which implies (3.22) by Toeplitz's Lemma. The proof is completed.

Remark 3.1. It is well known that NOD sequence contains independent random variable sequence and negatively associated random variable sequence as special cases. So the main results of the paper hold for them.

Remark 3.2. In Theorem 3.5, the condition $A_{n}=\sum_{j=1}^{n} a_{j} \uparrow \infty$ can be relaxed to $0<A_{n} \uparrow \infty$ when $1<r<2$. It suffices to prove (3.22). In fact, by (3.17) and (3.18), it follows that

$$
\begin{aligned}
& \sum_{k=1}^{\infty}\left|\frac{a_{k}(\log k) E Y_{k}}{A_{k}}\right| \\
& \leq \sum_{k=1}^{\infty} c_{k}^{-1}\left[c_{k} P\left(\left|X_{k}\right|>c_{k}\right)+E\left|X_{k}\right| I\left(\left|X_{k}\right|>c_{k}\right)\right] \\
& =\sum_{k=1}^{\infty} P\left(\left|X_{k}\right|>c_{k}\right)+\sum_{k=1}^{\infty} c_{k}^{-1} E\left|X_{k}\right| I\left(\left|X_{k}\right|>c_{k}\right) \\
& \leq C+\sum_{k=1}^{\infty} \sum_{c_{k} \leq j<c_{k}+1} c_{k}^{-1} E\left|X_{1}\right| I\left(\left|X_{1}\right|>c_{k}\right) \\
& \leq C+\sum_{j=1}^{\infty} \sum_{j-1<c_{k} \leq j} c_{k}^{-1} E\left|X_{1}\right| I\left(\left|X_{1}\right|>j-1\right) \\
& \leq C+C \sum_{j=2}^{\infty}(N(j)-N(j-1))(j-1)^{-1} \sum_{k=j-1}^{\infty} E\left|X_{1}\right| I\left(k<\left|X_{1}\right| \leq k+1\right) \\
& \leq C+C \sum_{k=1}^{\infty} \sum_{j=2}^{k+1} N(j)\left((j-1)^{-1}-j^{-1}\right) E\left|X_{1}\right| I\left(k<\left|X_{1}\right| \leq k+1\right) \\
& \leq C+C \sum_{k=1}^{\infty} \sum_{j=2}^{k+1} j^{r-2} E\left|X_{1}\right| I\left(k<\left|X_{1}\right| \leq k+1\right) \\
& \leq C+C \sum_{k=1}^{\infty} k^{r-1} E\left|X_{1}\right| I\left(k<\left|X_{1}\right| \leq k+1\right)
\end{aligned}
$$




$$
\leq C+C \sum_{k=1}^{\infty} E\left|X_{1}\right|^{r} I\left(k<\left|X_{1}\right| \leq k+1\right) \leq C+C E\left|X_{1}\right|^{r}<\infty
$$

\section{Exponential inequality for bounded NOD sequence}

It is well known that the exponential inequality plays an important role in various proofs of limit theorems. In particular, it provides a measure of convergence rate for the strong law of large numbers. In this section, we will establish an exponential inequality for NOD sequence, which can be applied to obtain the complete convergence and almost sure convergence for NOD sequence. In the following, we let $\left\{X_{n}, n \geq 1\right\}$ be a sequence of NOD random variables. Denote $B_{n}^{2}=\sum_{i=1}^{n} E X_{i}^{2}$ for each $n \geq 1$.

Theorem 4.1. Let $\left\{X_{n}, n \geq 1\right\}$ be a sequence of NOD random variables with $E X_{n}=0$ for each $n \geq 1$. If there exists a sequence of positive numbers $\left\{c_{n}, n \geq\right.$ $1\}$ such that $\left|X_{i}\right| \leq c_{n}$ for each $1 \leq i \leq n, n \geq 1$, then for any $t>0$ and any integer $n \geq 1$,

$$
E \exp \left\{t \sum_{i=1}^{n} X_{i}\right\} \leq \exp \left\{\frac{t^{2}}{2} e^{t c_{n}} \sum_{i=1}^{n} E X_{i}^{2}\right\}
$$

Proof. It is easy to check that for all $x \in \mathbf{R}$, the following inequality holds

$$
e^{x} \leq 1+x+\frac{1}{2} x^{2} e^{|x|}
$$

thus, by $E X_{i}=0$ and $\left|X_{i}\right| \leq c_{n}$ for each $1 \leq i \leq n, n \geq 1$, we have

$$
\begin{aligned}
E\left[e^{t X_{i}}\right] & \leq 1+t E X_{i}+\frac{1}{2} t^{2} E\left[X_{i}^{2} e^{t\left|X_{i}\right|}\right]=1+\frac{1}{2} t^{2} E\left[X_{i}^{2} e^{t\left|X_{i}\right|}\right] \\
& \leq 1+\frac{1}{2} t^{2} e^{t c_{n}} E X_{i}^{2} \leq \exp \left\{\frac{1}{2} t^{2} e^{t c_{n}} E X_{i}^{2}\right\}
\end{aligned}
$$

for any $t>0$. The last inequality above follows from the fact that $1+x \leq e^{x}$ for all $x \in \mathbf{R}$. By Lemma 1.1, Lemma 1.2 and (4.2), we can see that

$$
E \exp \left\{t \sum_{i=1}^{n} X_{i}\right\}=E\left\{\prod_{i=1}^{n} e^{t X_{i}}\right\} \leq \prod_{i=1}^{n} E e^{t X_{i}} \leq \exp \left\{\frac{t^{2}}{2} e^{t c_{n}} \sum_{i=1}^{n} E X_{i}^{2}\right\} .
$$

This completes the proof of the theorem.

Corollary 4.1. Let $\left\{X_{n}, n \geq 1\right\}$ be a sequence of NOD random variables such that $\left|X_{i}\right| \leq c_{n}$ for each $1 \leq \bar{i} \leq n, n \geq 1$, where $\left\{c_{n}, n \geq 1\right\}$ is a sequence of positive numbers. Then for any $t>0$ and any integer $n \geq 1$,

$$
E \exp \left\{t \sum_{i=1}^{n}\left(X_{i}-E X_{i}\right)\right\} \leq \exp \left\{\frac{t^{2}}{2} e^{2 t c_{n}} \sum_{i=1}^{n} E X_{i}^{2}\right\} \text {. }
$$


Theorem 4.2. Let $\left\{X_{n}, n \geq 1\right\}$ be a sequence of NOD random variables such that $\left|X_{i}\right| \leq c_{n}$ for each $1 \leq i \leq n, n \geq 1$, where $\left\{c_{n}, n \geq 1\right\}$ is a sequence of positive numbers. Then for any $\varepsilon>0$ such that $\varepsilon \leq e B_{n}^{2} /\left(2 c_{n}\right)$

$$
\begin{gathered}
P\left(\sum_{i=1}^{n}\left(X_{i}-E X_{i}\right) \geq \varepsilon\right) \leq \exp \left\{-\frac{\varepsilon^{2}}{2 e B_{n}^{2}}\right\}, \\
P\left(\sum_{i=1}^{n}\left(X_{i}-E X_{i}\right) \leq-\varepsilon\right) \leq \exp \left\{-\frac{\varepsilon^{2}}{2 e B_{n}^{2}}\right\}, \\
P\left(\left|\sum_{i=1}^{n}\left(X_{i}-E X_{i}\right)\right| \geq \varepsilon\right) \leq 2 \exp \left\{-\frac{\varepsilon^{2}}{2 e B_{n}^{2}}\right\} .
\end{gathered}
$$

Proof. By Markov's inequality and Corollary 4.1, we have that for any $t>0$,

$$
\begin{aligned}
P\left(\sum_{i=1}^{n}\left(X_{i}-E X_{i}\right) \geq \varepsilon\right) & =P\left(\exp \left\{t \sum_{i=1}^{n}\left(X_{i}-E X_{i}\right)\right\} \geq e^{t \varepsilon}\right) \\
& \leq e^{-t \varepsilon} E \exp \left\{t \sum_{i=1}^{n}\left(X_{i}-E X_{i}\right)\right\} \\
& \leq \exp \left\{-t \varepsilon+\frac{t^{2}}{2} e^{2 t c_{n}} B_{n}^{2}\right\} .
\end{aligned}
$$

Taking $t=\varepsilon /\left(e B_{n}^{2}\right)$, and noting that $2 t c_{n} \leq 1$, we can obtain (4.5). It is easily seen that $\left\{-X_{n}, n \geq 1\right\}$ is still a sequence of NOD random variables with $\left|-X_{i}\right| \leq c_{n}$ for each $1 \leq i \leq n, n \geq 1$, then it follows from (4.5) that

$$
\begin{aligned}
P\left(\sum_{i=1}^{n}\left(X_{i}-E X_{i}\right) \leq-\varepsilon\right) & =P\left(\sum_{i=1}^{n}\left(-X_{i}-E\left(-X_{i}\right)\right) \geq \varepsilon\right) \\
& \leq \exp \left\{-\frac{\varepsilon^{2}}{2 e B_{n}^{2}}\right\},
\end{aligned}
$$

which implies (4.6). Finally, (4.7) follows from (4.5) and (4.6) immediately. This completes the proof of the theorem.

Acknowledgements. The authors are most grateful to Managing Editor YoungJu Choie and anonymous referee for careful reading of the manuscript and valuable suggestions which helped to improve an earlier version of this paper.

\section{References}

[1] A. Adler, On the nonexistence of a law of the iterated logarithm for weighted sums of identically distributed random variables, J. Appl. Math. Stochastic Anal. 3 (1990), no. $2,135-140$.

[2] R. G. Antonini, J. S. Kwon, S. H. Sung, and Andrei I. Volodin, On the strong convergence of weighted sums, Stochastic Anal. Appl. 19 (2001), no. 6, 903-909. 
[3] A. Bozorgnia, R. F. Patterson, and R. L. Taylor, Limit theorems for dependent random variables, World Congress of Nonlinear Analysts '92, Vol. I-IV (Tampa, FL, 1992), 16391650, de Gruyter, Berlin, 1996

[4] Y. S. Chow and T. L. Lai, Limiting behavior of weighted sums of independent random variables, Ann. Probability 1 (1973), no. 5, 810-824.

[5] V. Fakoor and H. A. Azarnoosh, Probability inequalities for sums of negatively dependent random variables, Pakistan J. Statist. 21 (2005), no. 3, 257-264.

[6] K. Joag-Dev and F. Proschan, Negative association of random variables with applications, Ann. Statist. 11 (1983), no. 1, 286-295.

[7] H. C. Kim, The Hájek-Rényi inequality for weighted sums of negatively orthant dependent random variables, Int. J. Contemp. Math. Sci. 1 (2006), no. 5-8, 297-303.

[8] M.-H. Ko, K.-H. Han, and T.-S. Kim, Strong laws of large numbers for weighted sums of negatively dependent random variables, J. Korean Math. Soc. 43 (2006), no. 6, 13251338.

[9] M.-H. Ko and T.-S. Kim, Almost sure convergence for weighted sums of negatively orthant dependent random variables, J. Korean Math. Soc. 42 (2005), no. 5, 949-957.

[10] Q. Wu, Complete convergence for negatively dependent sequences of random variables, J. Inequal. Appl. 2010, Art. ID 507293, 10 pp.; doi:10.1155/2010/507293.

[11] W. Wu, On the strong convergence of a weighted sum, Statist. Probab. Lett. 44 (1999), no. $1,19-22$.

Xuejun Wang

School of Mathematical Science

ANHUi UNIVERSITY

Hefei 230039, P. R. China

E-mail address: wxjahdx2000@126.com

Shuhe Hu

School of Mathematical Science

Anhui University

Hefei 230039, P. R. China

E-mail address: hushuhe@263.net

ANDREI I. VOLODIN

Department of Mathematics and Statistics

UNIVERSITY OF REGINA

Regina, CANAdA

E-mail address: volodin@math.uregina.ca 